\title{
Embryos and ancestors: Reconstructing gene regulatory networks and embryonic development in ancestral echinoids
}

\author{
Eric M Erkenbrack \\ Yale University \\ Jeffrey R Thompson \\ USC \\ September 25, 2016 \\ GSA - T140
}




\section{EMBRYOS}

AND

\section{ANCESTORS}

By

SIR GAVIN DE BEER, F.R.S.
"Embryos undergo development; ancestors have undergone development, but in their day they also were the products of development."

p. 1 (1940) 


\section{Embryos and ancestors}
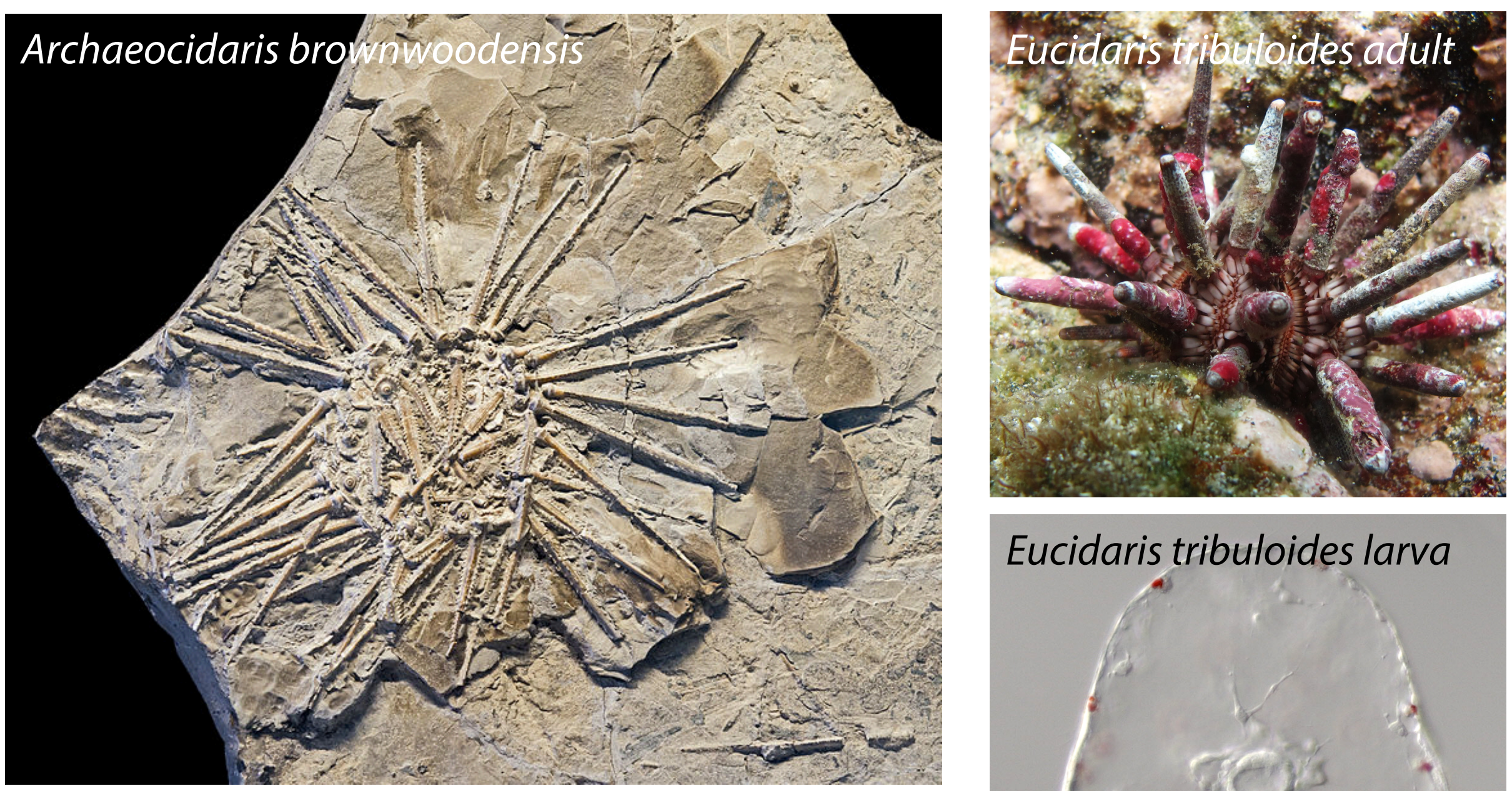

Eucidaris tribuloides larva

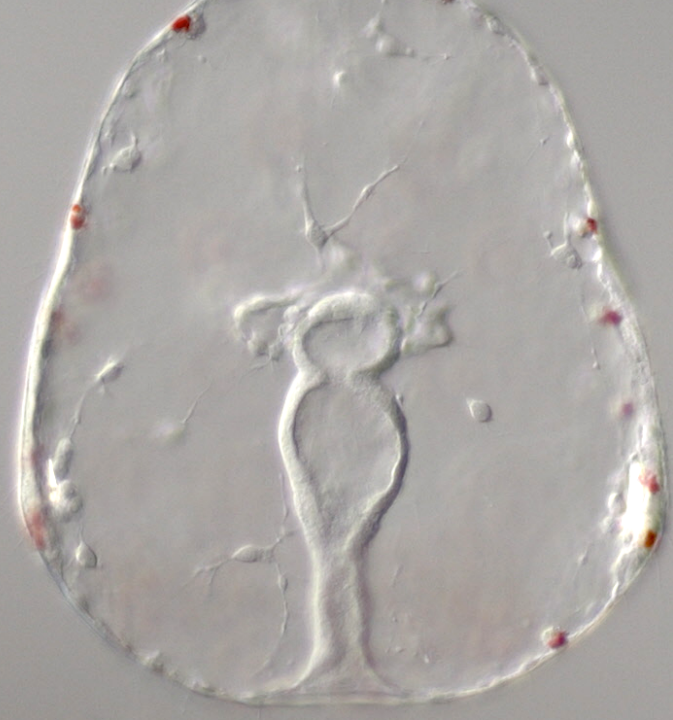




\section{Motivating questions}

- What can we say about the embryos and developmental programs of Archaeocidaris spp.?

- What kinds of data are at our disposal?

- Can we simultaneously investigate fundamental processes of development and evolution along the way? 


\section{Phylogeny of echinoids}

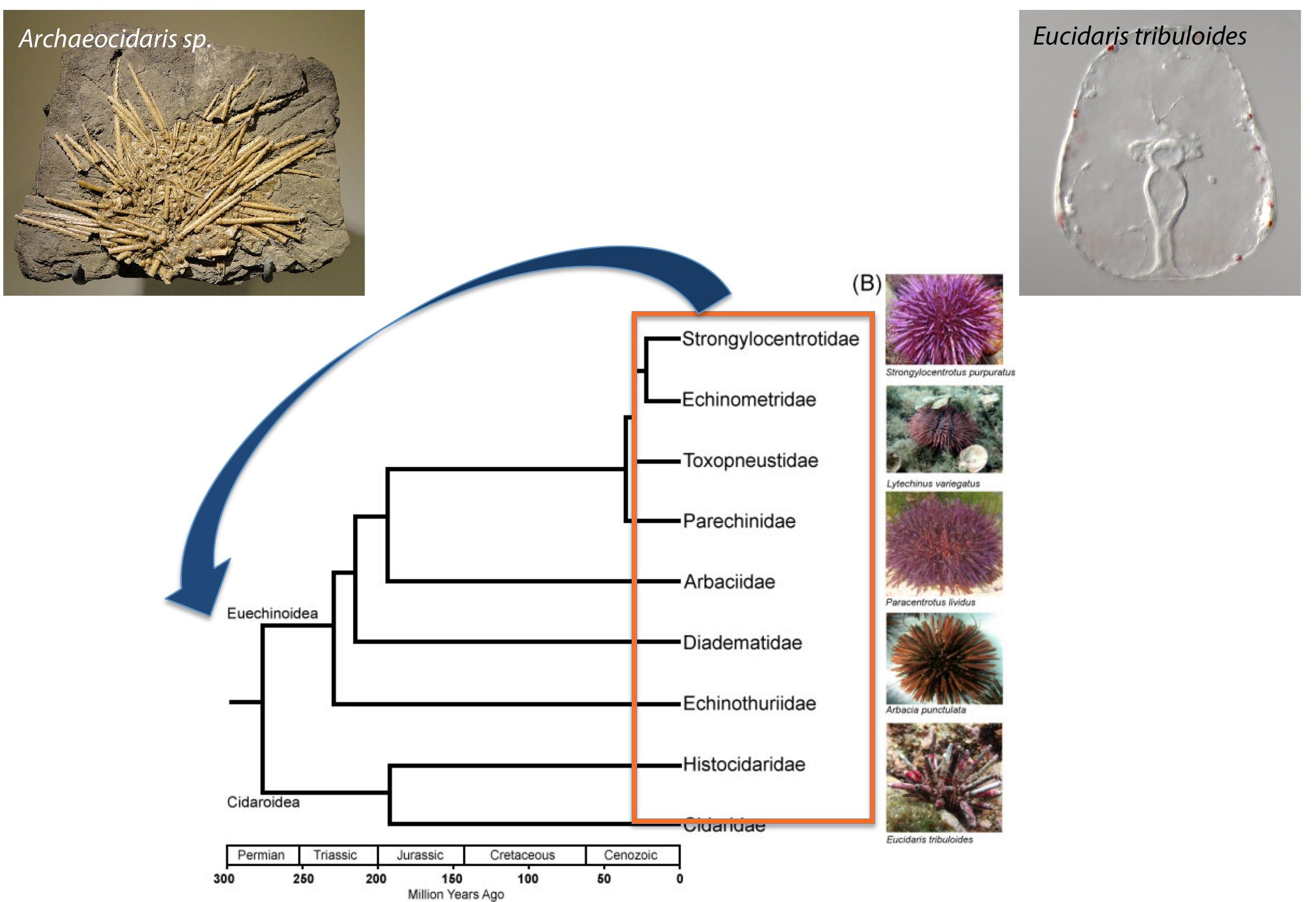




\section{What kinds of data can we collect to make inferences about ancestral embryos?}

- Developmental modes

- larval life strategies, e.g. direct v. indirect development

- Cell lineages

- clade-specific novelties, e.g. larval skeleton, larval pigment, etc.

- Gene families

- Gene loss, sub- and neo-functionalization of duplicated genes, etc.

- Gene regulation

- Gene regulatory networks (linkages, circuits), regulatory states (spatial localization of gene products), etc.

$\rightarrow$ Common to all of these datasets is the importance of sampling numerous taxa 


\section{What is a gene regulatory network (GRN)?}

\section{Gene Regulation}

Protein products of genes (e.g., transcription factors, signaling cascades) regulate the production (transcription) of other genes

Gene Regulatory Network

A wiring diagram representing the intricate, recursive regulatory circuitry of gene/protein interactions in the cell (or the embryo)

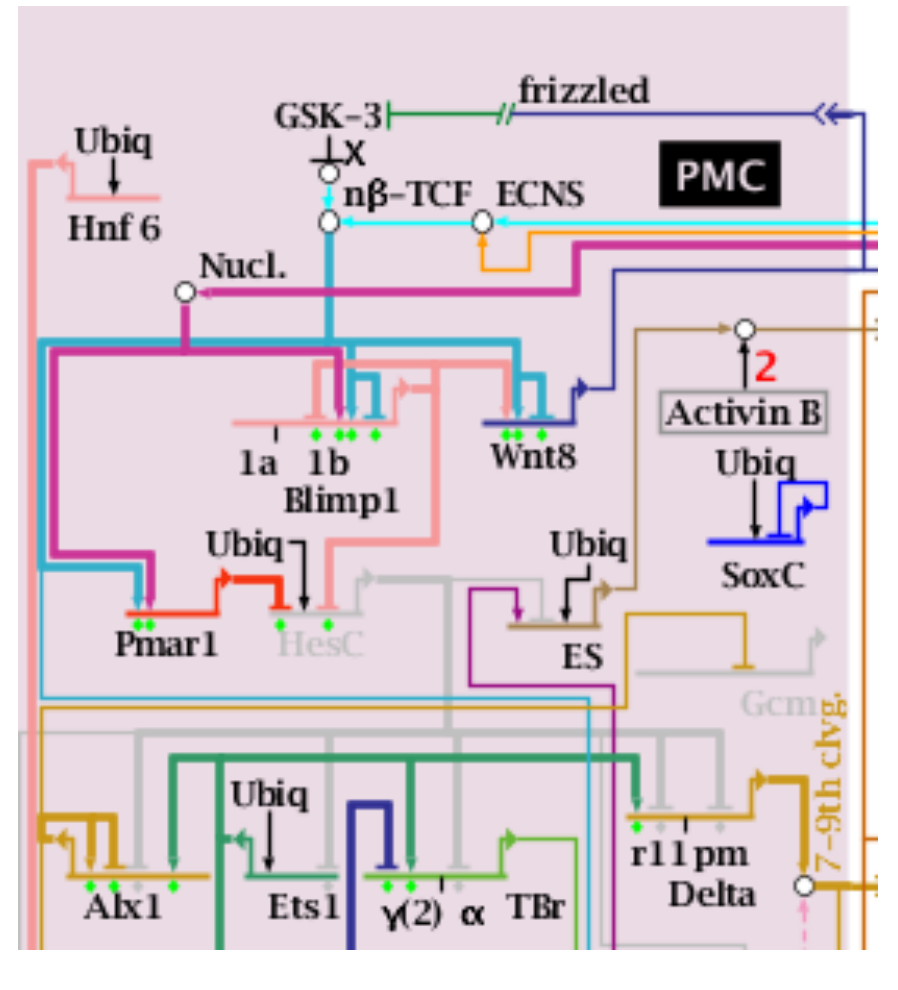




\section{What is a regulatory state (RS)?}

\section{Regulatory State}

-- The spatial output of gene regulatory networks in the embryo

-- The total set of regulatory genes present in a given cell

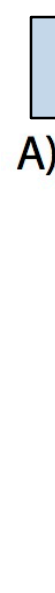

B)

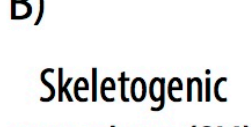
mesoderm (SM)

Non-skeletogenic mesoderm (NSM)

Veg2/Anterior Endoderm (AE)

Veg1/Posterior Endoderm (PE) Perianal Ectoderm

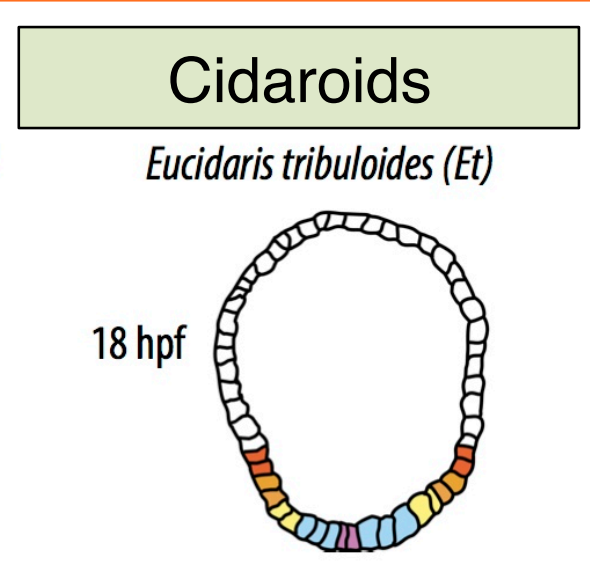

Sp Regulatory Common Regulatory Et Regulatory

\begin{tabular}{|l|l|l|}
\hline \multicolumn{1}{|l}{ State } & \multicolumn{1}{l}{ State } & \multicolumn{1}{c}{ State } \\
\hline ets $1 / 2$ & alx 1 & delta \\
\hline
\end{tabular}

\begin{tabular}{|c|c|} 
myc, \\
gcm, gatae
\end{tabular} ets $1 / 2$

gatae, blimp1, foxa, myc $\operatorname{hox} 11 / 13 b$

bra, hox11/13b, eve, wnt8 eve, wnt8 


\section{Strongylocentrotus purpuratus Global GRN, 18-30 hpf (hours post fertilization)}
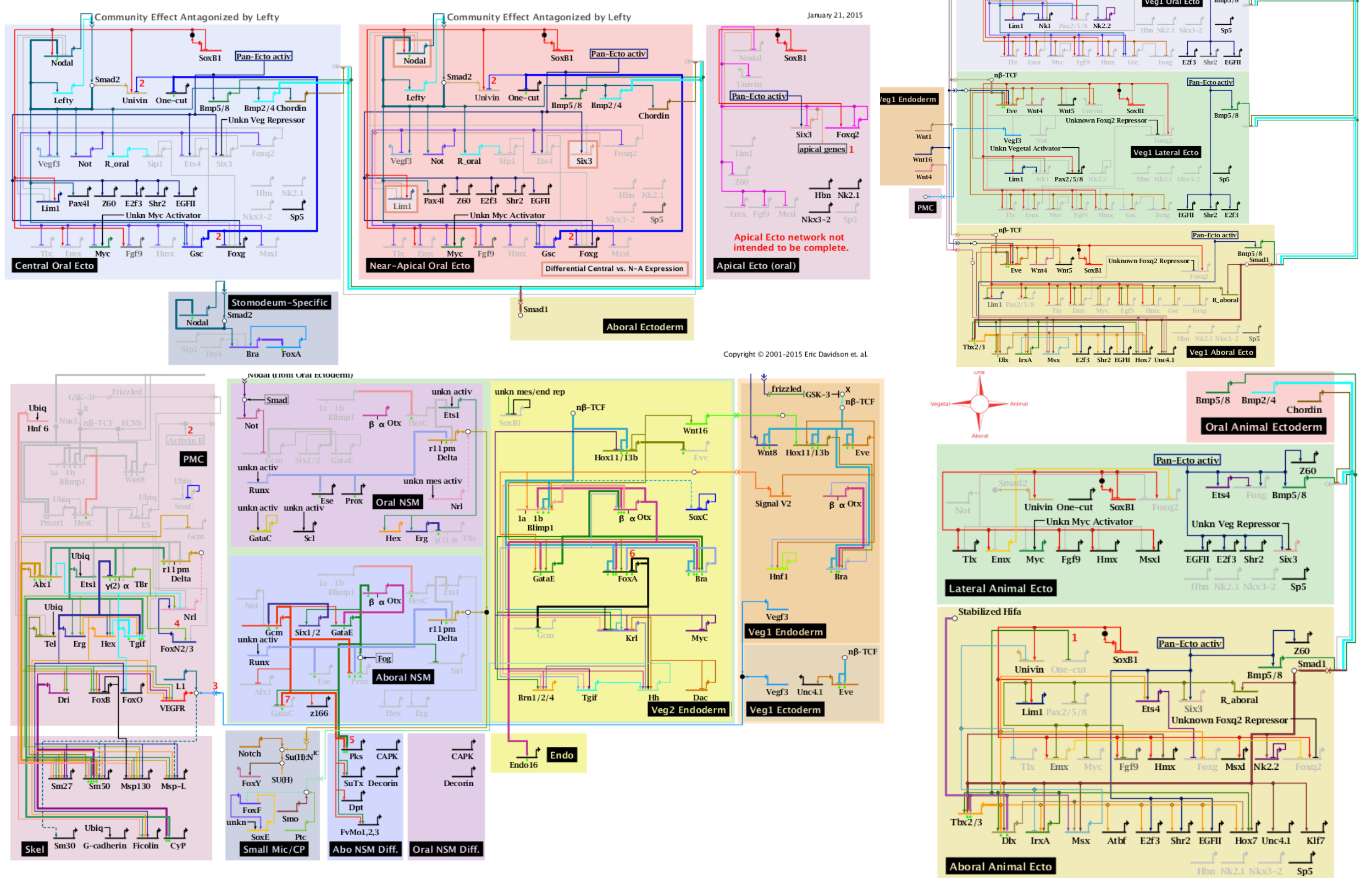


\section{Comparative analyses in echinoderms}

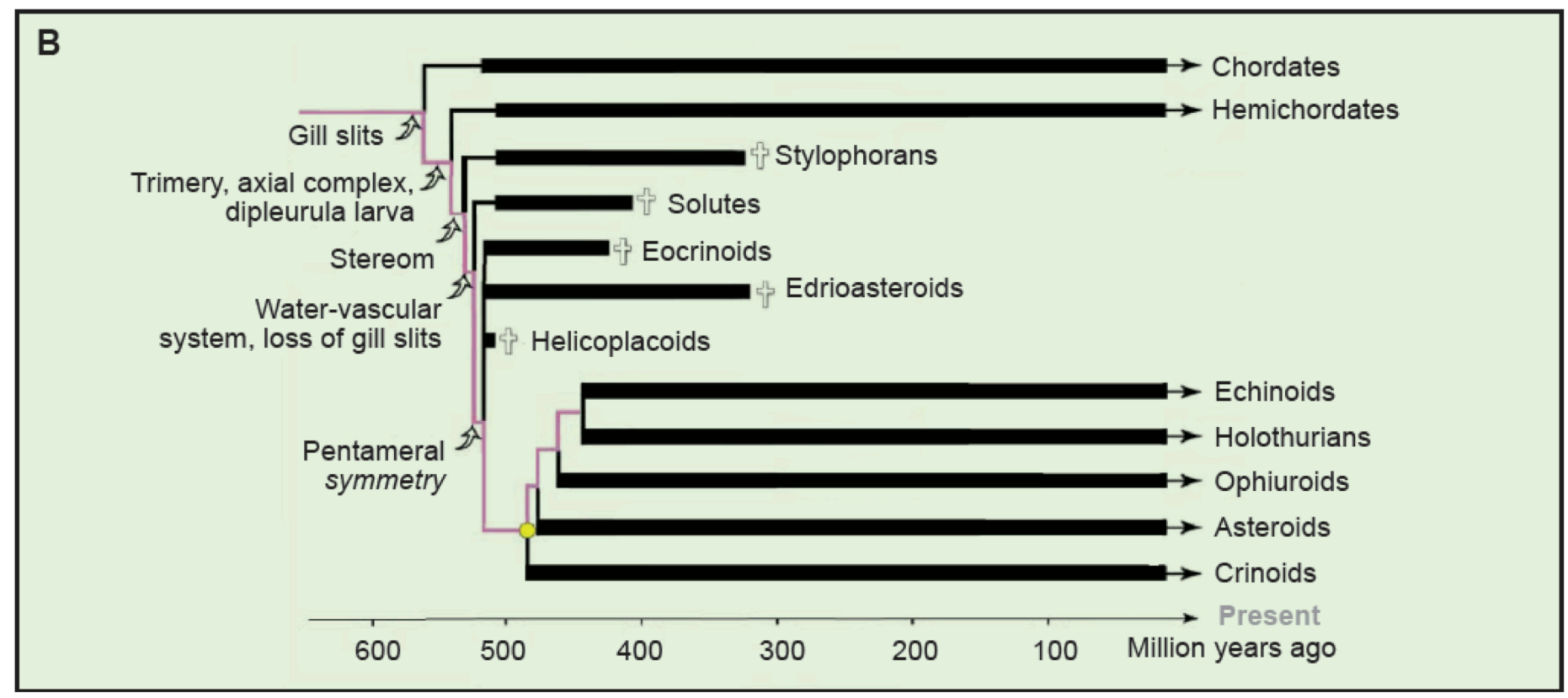

Bottjer et al. (2005)

$\rightarrow$ Embryonic development of echinoid outgroups, e.g. asteroids, ophiuroids, holothuroids, afford polarity of developmental programs and modes 


\section{A good fossil record is a developmental evolutionary biologist's best friend}

Accurate dating of echinoderm fossils combined with developmental studies of modern descendants reveals the tempo and mode of developmental evolution

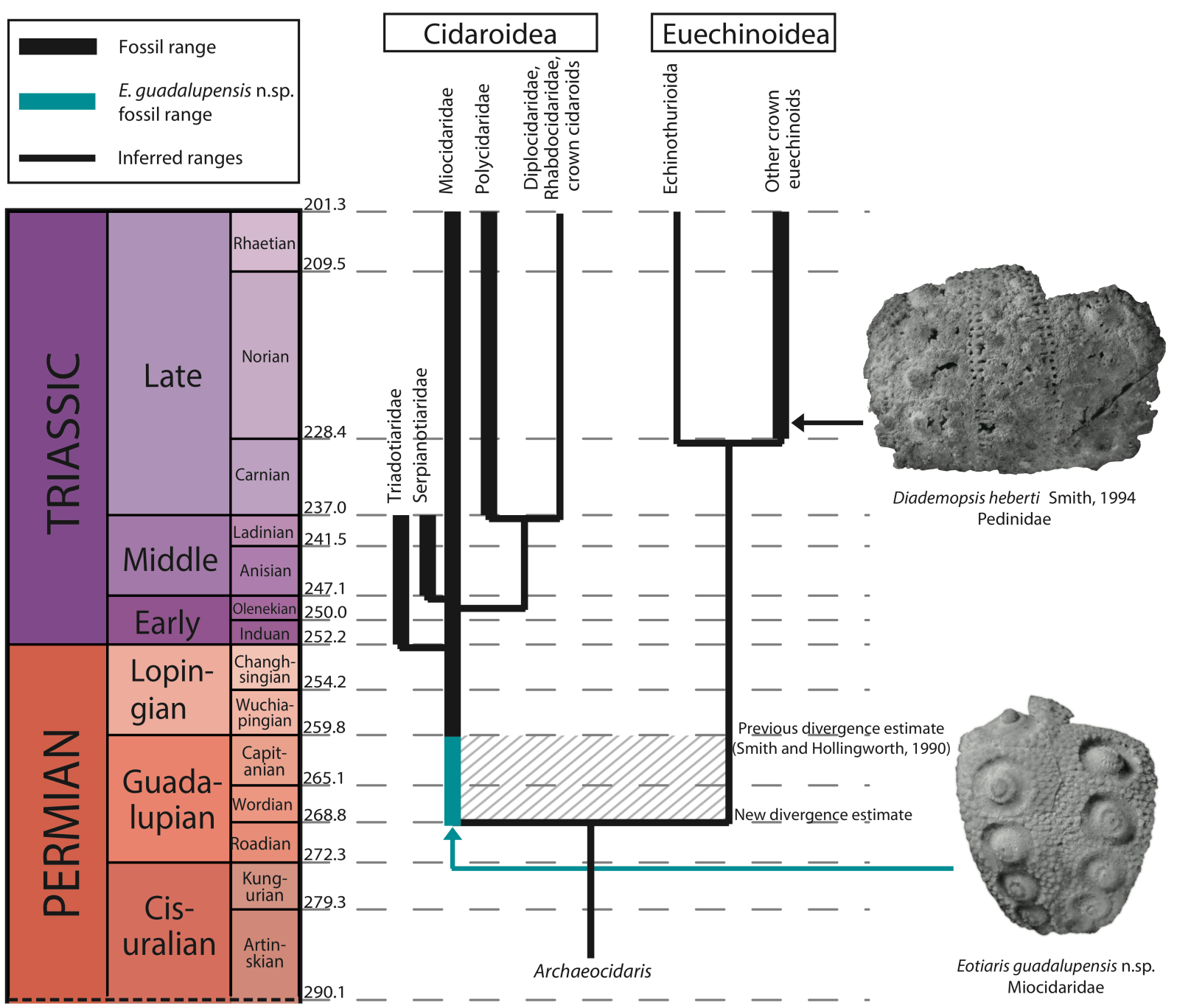




\section{Expression of mesoderm-specific regulatory gene tbrain in echinoderms}

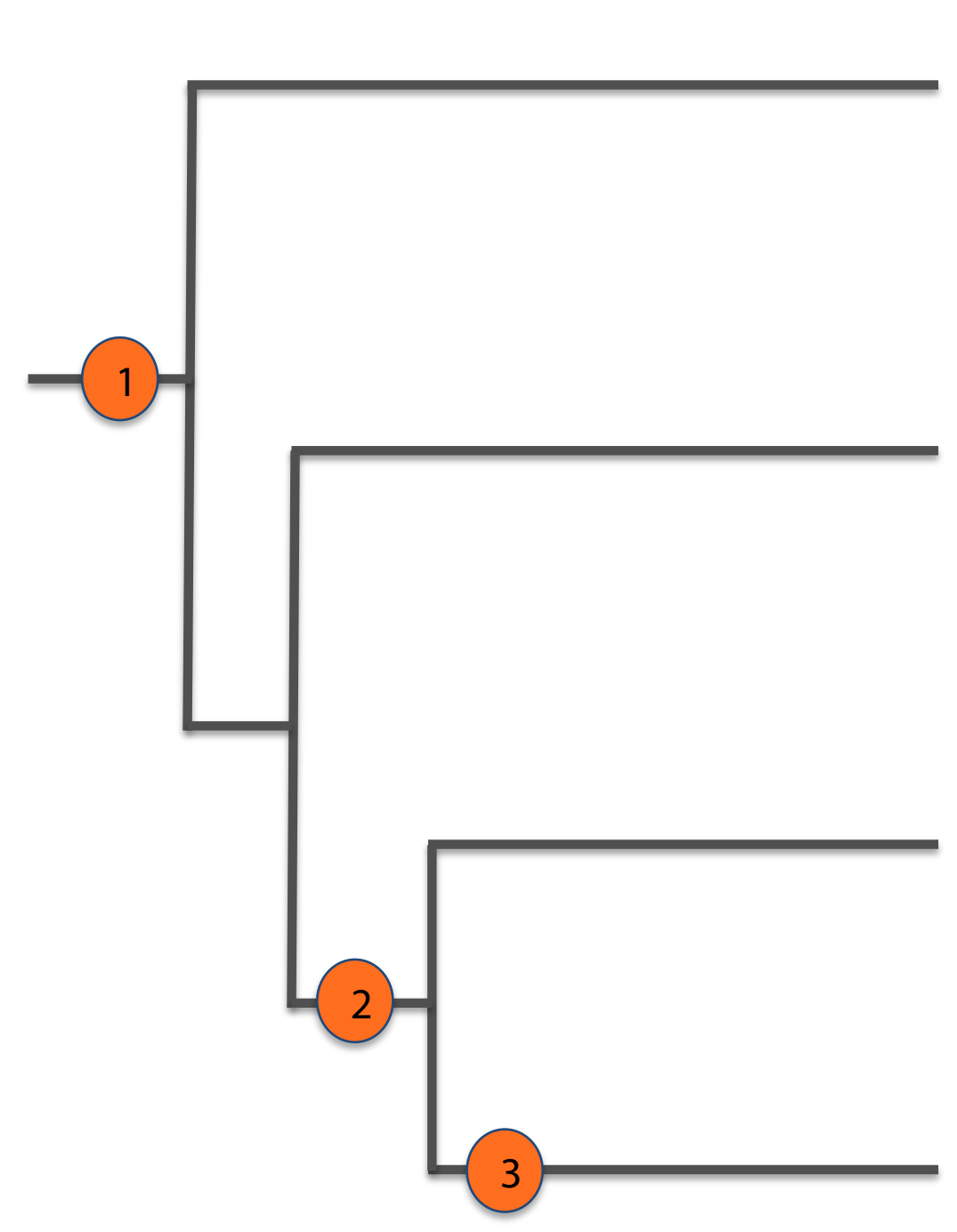

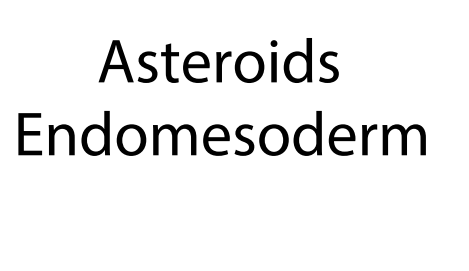

Holothuroids: Endomesoderm

Cidaroids: Mesoderm

Euechinoids: Skeletogenic Mesoderm

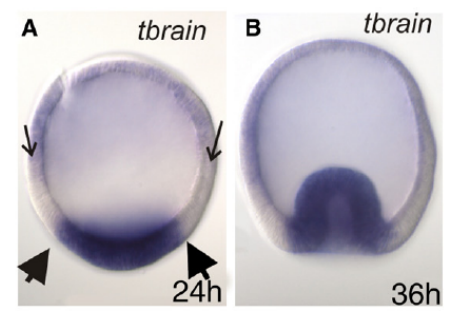

(Hinman and Davidson, 2007)
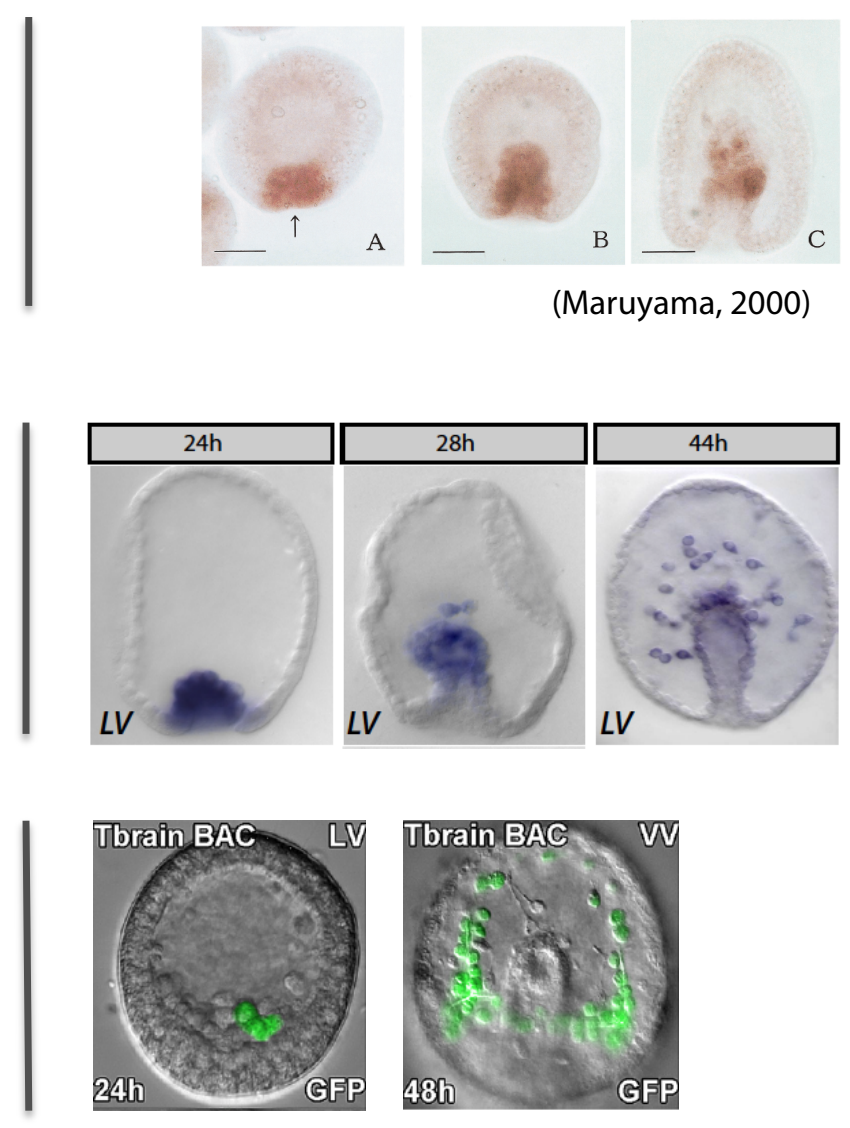

(Wahl et al, 2009) 


\section{Dating deep-time developmental programs}

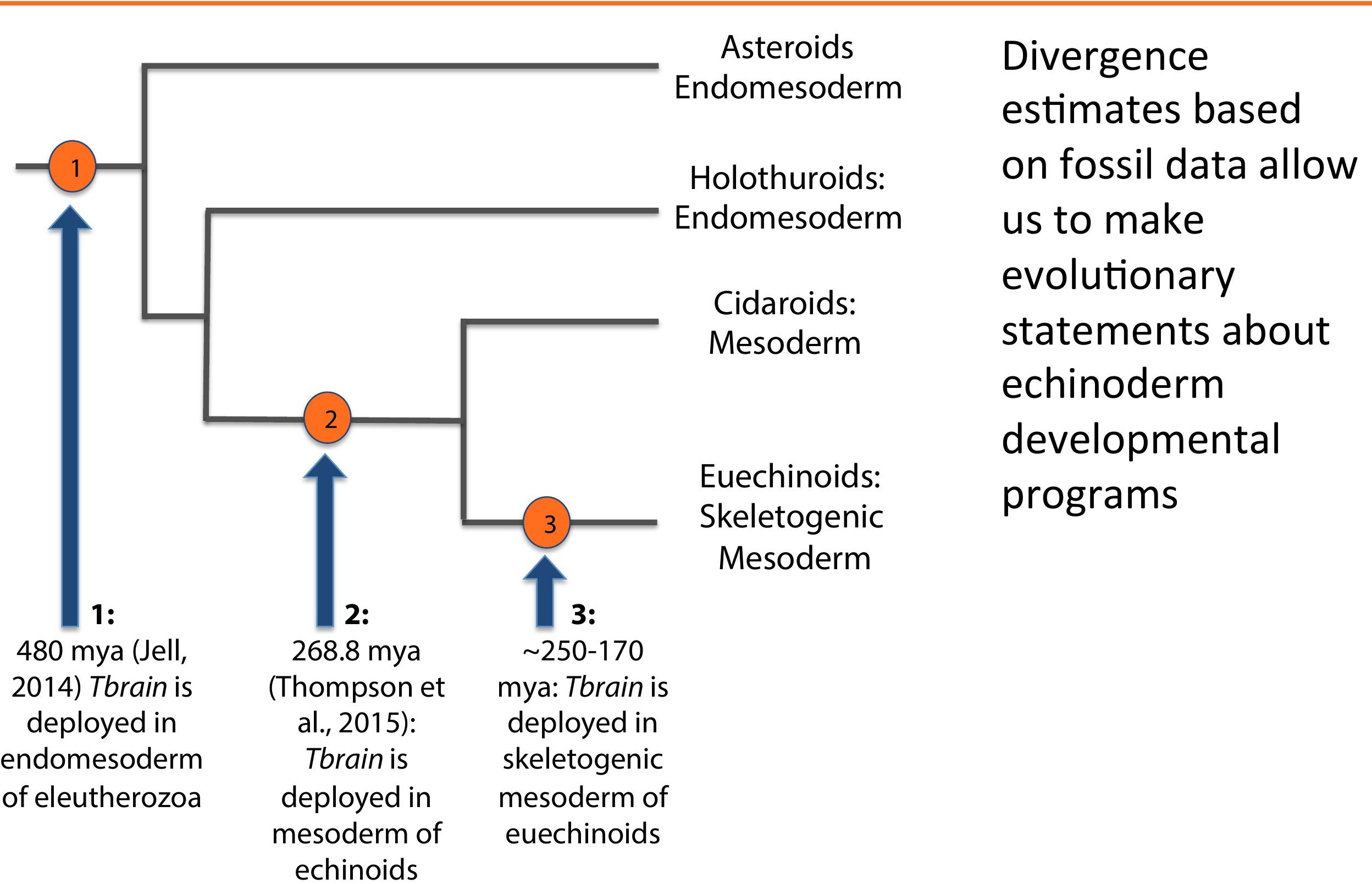




\section{A highly conserved mesodermal regulatory state in echinoderms}

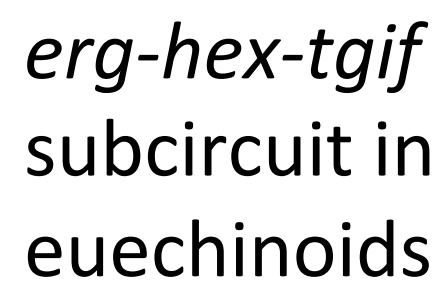

erg-hex-tgif

subcircuit in

euechinoids

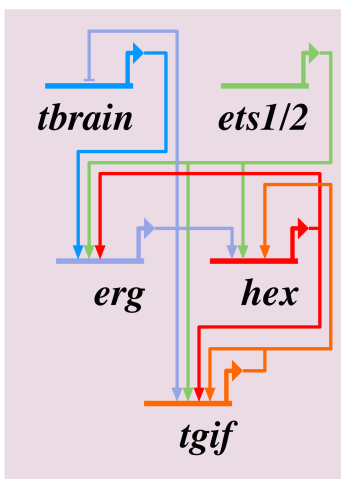

PMCs

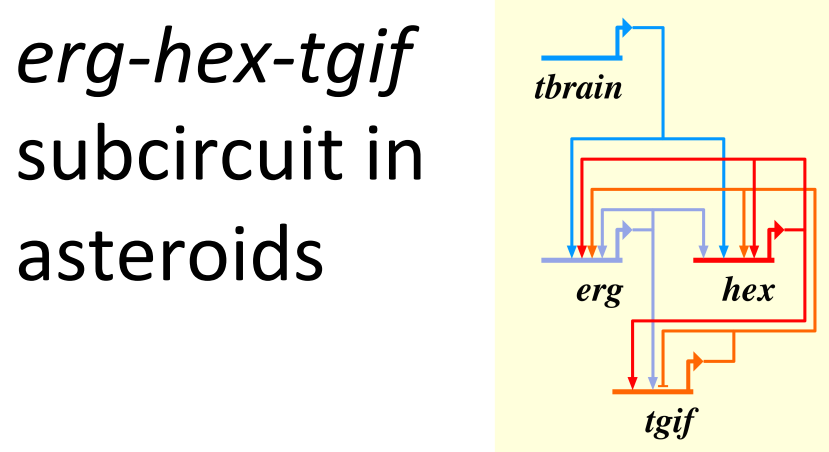

Endomesoderm

erg-hex-tgif subcircuit in cidaroids

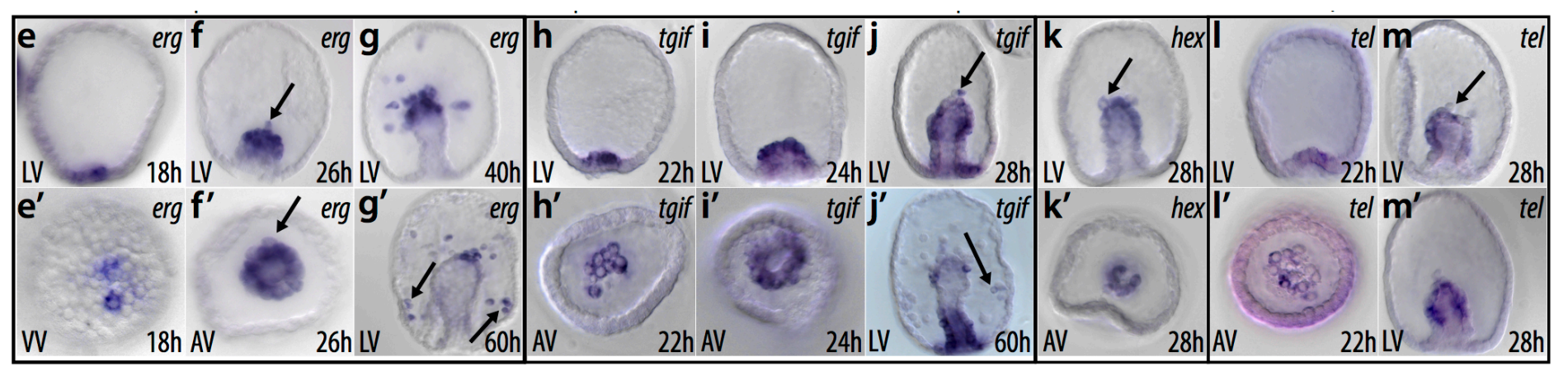

Erkenbrack et al. (2016) Dev Gen Evo 


\section{Reconstructing ancestral regulatory states in echinoderms and dating their appearance}

Table 4 Ancestral state reconstruction for embryos of ancestors of extant echinoderm clades by comparative analysis of spatial gene expression data from three or more taxa

At least 481 mya: in ancestral embryos prior to the asterozoanechinozoan divergence

1. erg was a mesodermal driver at blastula stage and gastrula stage

2. hex was a mesodermal driver at blastula stage

3. tgif was a mesodermal driver at blastula stage and gastrula stage

4. tgif was an endodermal driver at mid-gastrula

5. erg-hex-tgif kernel operated in mesoderm

6. Prediction: hex is likely to be expressed in mesoderm of

holothurians, but endodemal expression after blastula stage is unclear

At least 462 mya: in ancestral embryos prior to the holothuroid-echinoid divergence

7. erg and tgif were initiated in the mesoderm and tgif came to be expressed in the endoderm at a later time in development; whereas erg remained restricted to the mesoderm throughout early embryonic development to fulfill its ancestral function, tgif was expressed first in the mesoderm and then in the mesoderm and the endoderm

8. tgif mesoderm expression at mid-gastrula stage was either lost in asteroids or gained in the lineage leading to the last common ancestor of echinozoans

9. erg was expressed in the skeletogenic lineage at least as late in development as mid-gastrula stage

10. hex endodermal expression is acquired early in asteroid embryogenesis or lost in last common ancestor of extant echinozoans

At least 268 mya: in ancestral embryos at the cidaroid-euechinoid divergence, e.g., in Archaeocidaris embryos

11. erg, hex, and tel were initiated in a few cells at the center of the vegetal pole; later in the lineage leading to camaradont euechinoids following the cidaroid-euechinoid divergence, these three genes are restricted PMCs prior to PMC ingression

12. tgif remains expressed in mesodermal cells that ingressed into the blastocoel (tgif is not expressed in mesodermal cells that have ingressed in holothuroids)

a

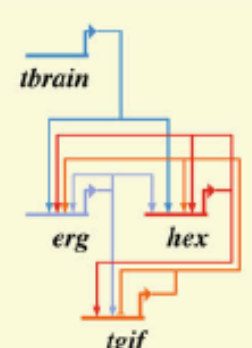

tgif

Endomesoderm

b

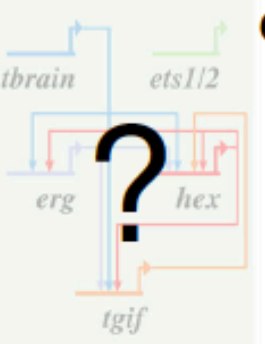

C $\overline{\text { tbrain }}$ etsI/2

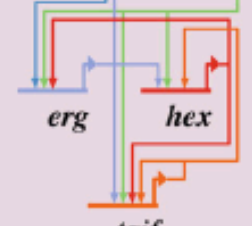

tgif

PMCs

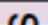

Q

d

mya/

Era

$\frac{0}{\frac{0}{0}}$

음

일

1

481

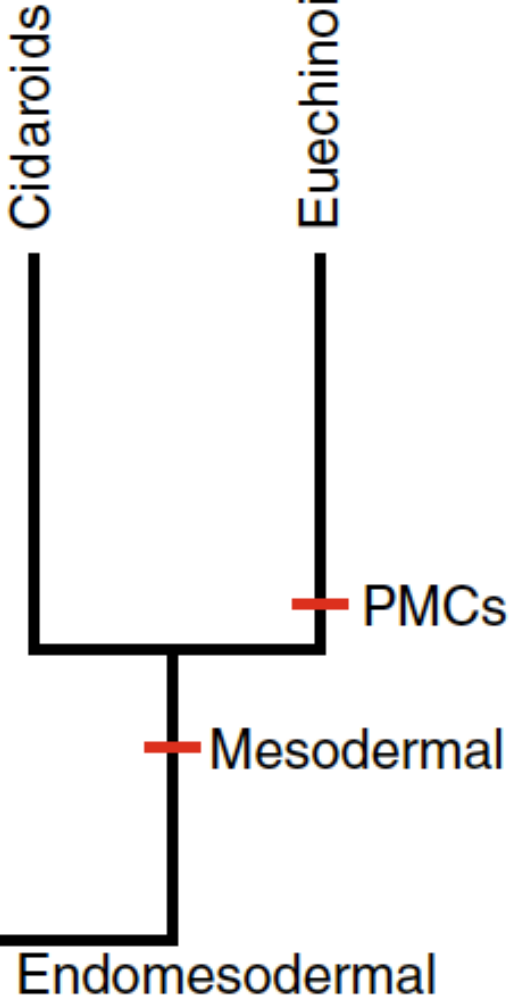

Erkenbrack et al. (2016) Dev Gen Evo

Erkenbrack et al. (2016) Dev Gen Evo 


\section{Going forward}

\section{Conceptual Toolkit}

Integrating paleontological data and embryonic developmental data informs assumptions regarding the genomic and morphological alterations that must have occurred in lineages leading to modern taxa

Evolution of developmental programs

Interdisciplinary studies reveal the tempo and mode of evolution of genomically encoded developmental programs

More taxa, fewer problems

Comparative analyses of embryonic development and omics data of numerous taxa afford triangulation of evolutionary inferences and ancestral state reconstruction 


\section{Acknowledgments}

Eric H Davidson

(DNA/RNA/Embryos/NO-BS)

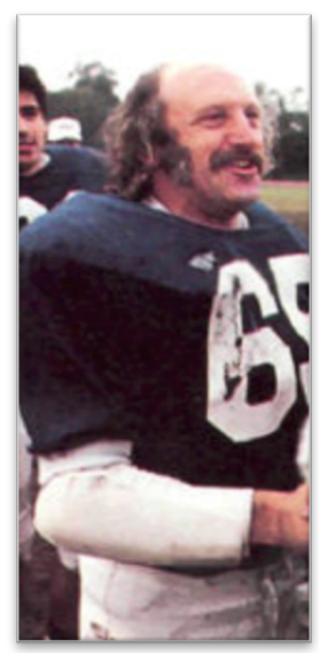

Dave Bottjer

(Fossils/Paleogenomics/Psych)
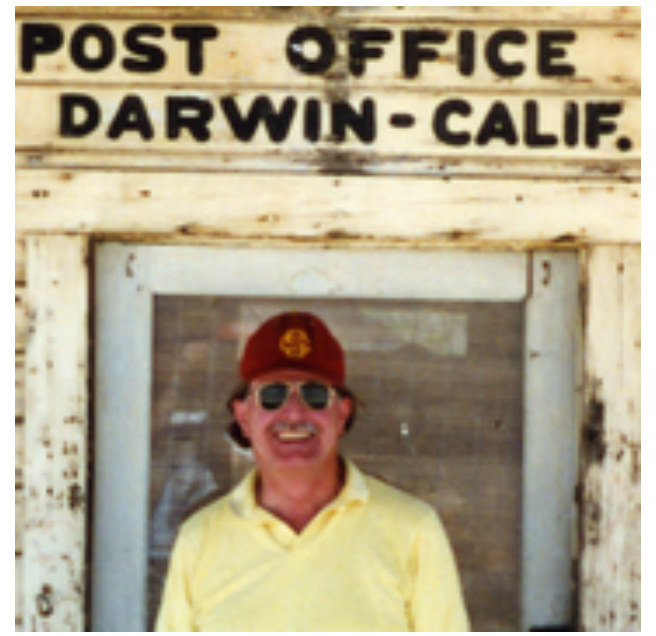

Günter P Wagner

(DNA/RNA/Protein/Cells/Guns)

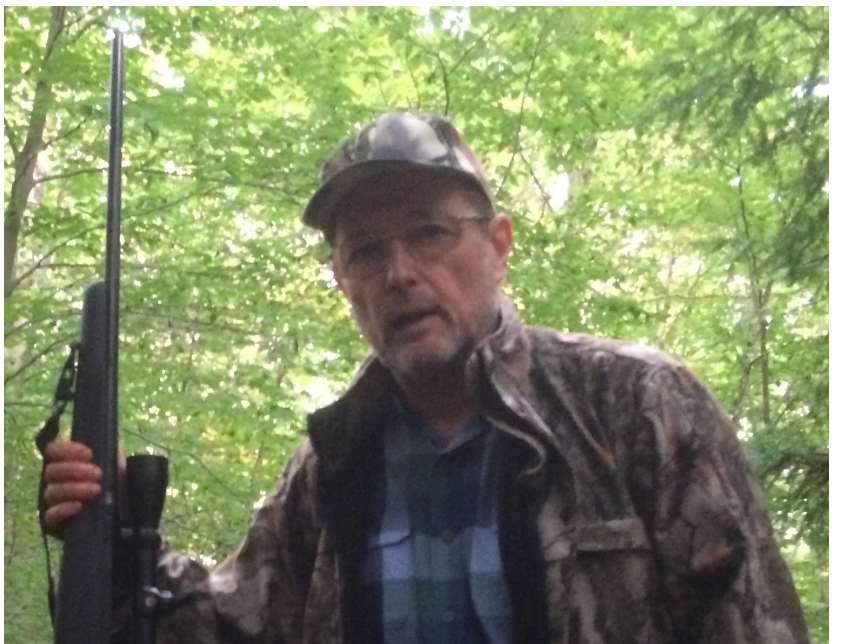

Archaeocidaris Dream Team Jeffrey R Thompson Liz Petsios

Feng Gao

Davidson Lab Members

Laura Romano (Denison)

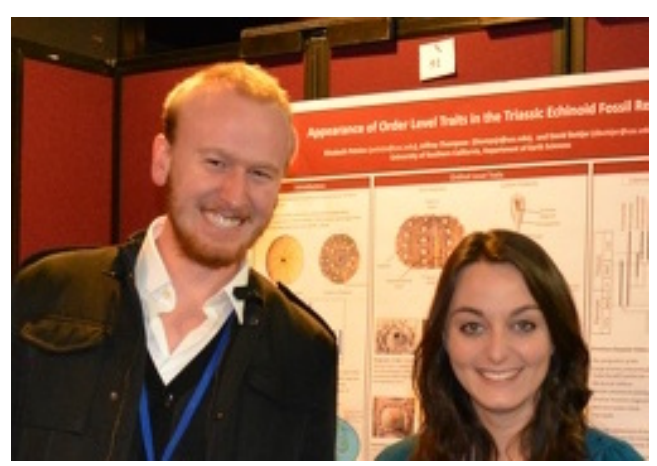

Funding

This work was funded by NSF CREATIV \#1240626

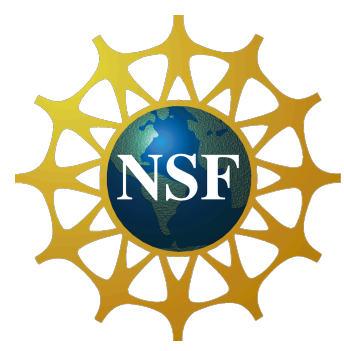




\section{WARNING}

\section{Interdisciplinary Advertisement}

I encourage paleontologists in the audience to reach out to developmental biologists! 


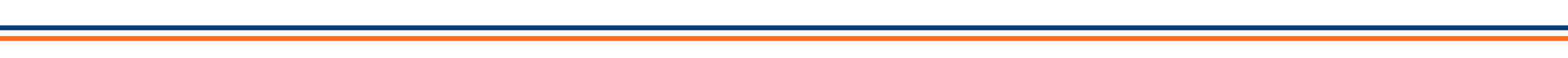




\section{Evolutionary inferences of the appearance of GRN circuitry}

Comparative analyses of gene expression and knowledge of the fossil record revealed probability metrics for the appearance this developmental program

A

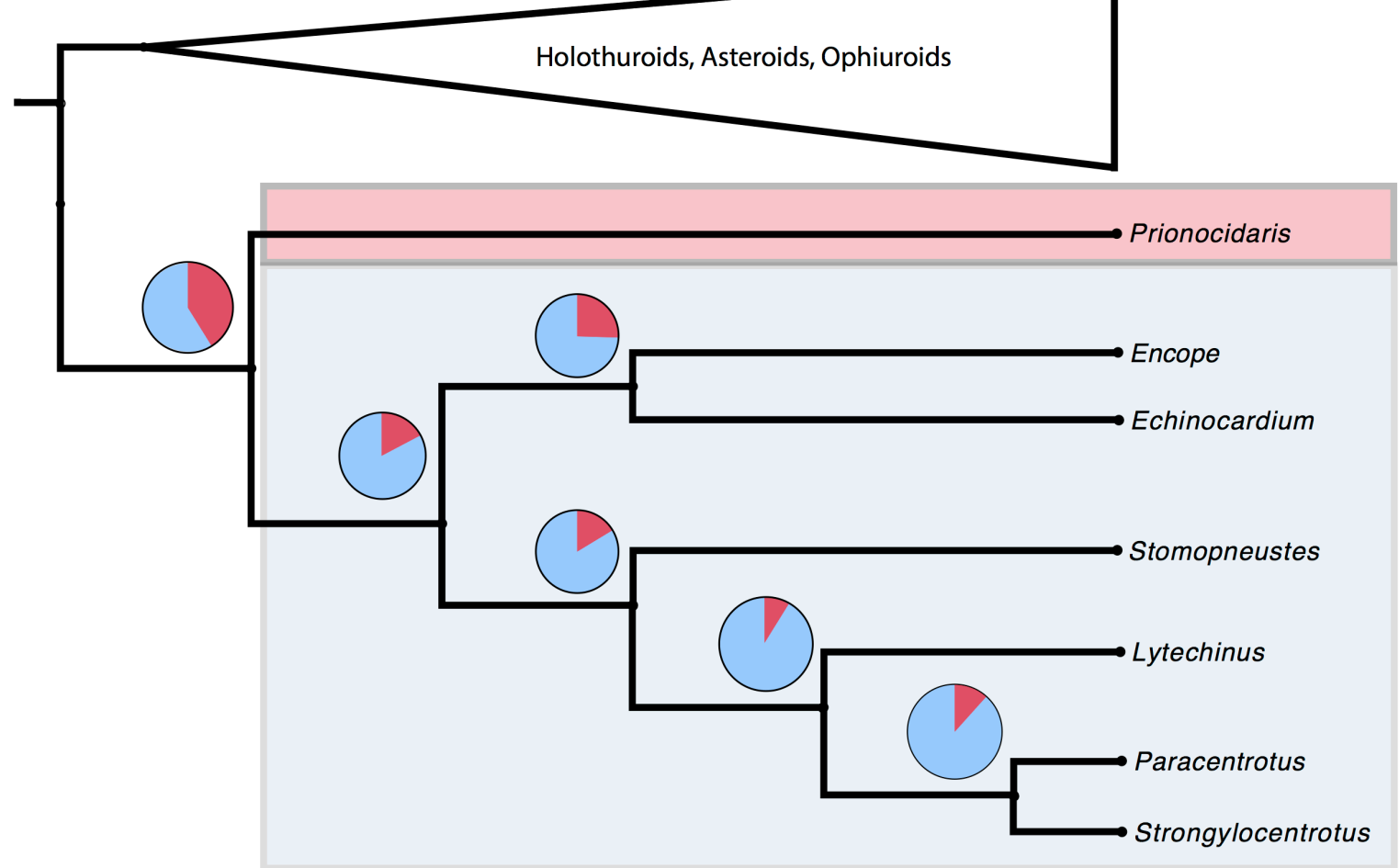

B Cidaroid Skeletogenic Micromere-descendants

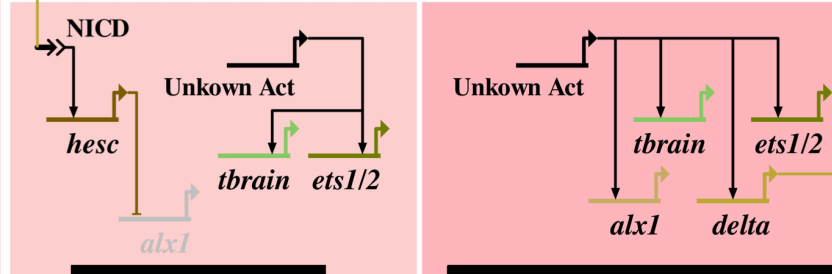

Adjacent cells

Micromere-descendants

C

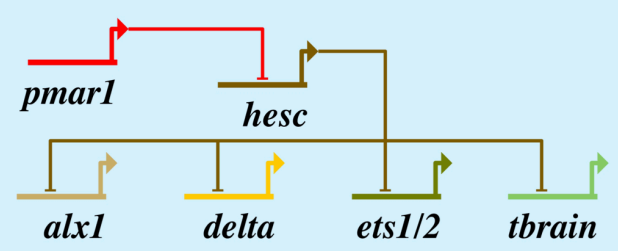

Micromeres

Thompson, Erkenbrack, et al. (In review) PNAS 